\title{
openheart Clinically feasible stratification of 1-year to 3-year post-myocardial infarction risk
}

\author{
Benjamin D Horne, ${ }^{1,2}$ Joseph B Muhlestein, ${ }^{1,3}$ Durgesh Bhandary, ${ }^{4}$ \\ Greta L Hoetzer, ${ }^{4}$ Naeem D Khan, ${ }^{4}$ Tami L Bair, ${ }^{1}$ Donald L Lappé ${ }^{1,3}$
}

\begin{abstract}
- Additional material is published online only. To view please visit the journal online (http://dx.doi.org/10.1136/ openhrt-2017-000723).
\end{abstract}

To cite: Horne BD, Muhlestein JB, Bhandary D, et al. Clinically feasible stratification of 1-year to 3-year post-myocardial infarction risk. Open Heart 2018;5:e000723. doi:10.1136/ openhrt-2017-000723

Received 11 September 2017 Revised 1 December 2017 Accepted 29 December 2017
Check for updates

${ }^{1}$ Intermountain Heart Institute, Intermountain Medical Center, Salt Lake City, Utah, USA ${ }^{2}$ Department of Biomedical Informatics, University of Utah, Salt Lake City, Utah, USA ${ }^{3}$ Cardiology Division, Department of Internal Medicine, University of Utah, Salt Lake City, Utah, USA

${ }^{4}$ AstraZeneca Pharmaceuticals LP, Wilmington, Delaware, USA

\section{Correspondence to}

Dr Benjamin D Horne; benjamin. horne@imail.org

\section{ABSTRACT}

Objective Post-myocardial infarction (MI) care is crucial to preventing recurrent major adverse cardiovascular events (MACE), but can be complicated to personalise. A tool is needed that effectively stratifies risk of cardiovascular (CV) events 1-3 years after Ml but is also clinically usable. Methods Patients surviving $\geq 1$ year after an index Ml with $\geq 1$ risk factor for recurrent Ml (ie, age $\geq 65$ years, prior MI, multivessel coronary disease, diabetes, glomerular filtration rate $<60 \mathrm{~mL} / \mathrm{min} / 1.73 \mathrm{~m}^{2}$ ) were studied. Cox regression derived sex-specific Intermountain Major Adverse Cardiovascular Events (IMACE) risk scores for the composite of 1-year to 3-year MACE (CV death, MI or stroke). Derivation was performed in $70 \%$ of subjects ( $n=1342$ women; 3047 men), with validation in the other $30 \%$ ( $n=576$ women; 1290 men). Secondary validations were also performed.

Results In women, predictors of CV events were glucose, creatinine, haemoglobin, platelet count, red cell distribution width (RDW), age and B-type natriuretic peptide (BNP); among men, they were potassium, glucose, blood urea nitrogen, haematocrit, white blood cell count, RDW, mean platelet volume, age and BNP. In the primary validation, in women, IMACE ranged from 0 to 11 (maximum possible: 12) and had $\mathrm{HR}=1.44$ per +1 score (95\% $\mathrm{Cl} 1.29$ to $1.61 ; \mathrm{P}<0.001)$; men had IMACE range 0-14 (maximum: 16$)$ and $\mathrm{HR}=1.29$ per +1 score $(95 \% \mathrm{Cl}$ 1.20 to 1.38; $P<0.001$ ). IMACE $\geq 5$ in women ( $\geq 6$ in men) showed strikingly higher MACE risk.

Conclusions Sex-specific risk scores strongly stratified 1-year to 3-year post-MI MACE risk. IMACE is an inexpensive, dynamic, electronically delivered tool for evaluating and better managing post-Ml patient care.

\section{INTRODUCTION}

Diagnostic and prognostic risk prediction tools are often created with the goal to improve medical care quality. ${ }^{1-8}$ In patients with a history of myocardial infarction (MI), the quantification and stratification of risk for future major adverse cardiovascular events (MACE) are challenging, in part because most risk tools use predictors or approaches that add time to a clinician's workload. Various such tools apply to prognostication after acute coronary syndrome. ${ }^{36-11}$ With the recent approval of dual antiplatelet therapy

\section{Key questions}

What is already known about this subject?

- Long-term use of dual antiplatelet therapy and other post-myocardial infarction (MI) care is effective at reducing major adverse cardiovascular events (MACE), but limiting risks and costs are increasingly a concern.

What does this study add?

- Developed on the methodologies of successfully implemented dynamic clinical risk scores, the sex-specific Intermountain Major Adverse Cardiovascular Events (IMACE) models have a greater ability than other clinical decision tools in predicting 3-year MACE and are more practical to deliver and efficiently use in clinical practice.

How might this impact on clinical practice?

- Clinicians may receive IMACE and other post-MI risk prediction scores within the clinical workflow via the electronic health record without changing their practice environment or processes, and use IMACE to direct their use of time and other resources towards patients in need of more precise evaluation and care.

(DAPT) for treatment periods longer than 12 months following acute MI, ${ }^{12-16}$ the ability to risk-stratify patients early in the care process to better personalise care plans would be beneficial. ${ }^{17}$

Use of risk tools to guide clinical care following an acute MI may involve higher precision in tailoring the prescription of antiplatelet agents and other medications to a patient's needs, as well as gauging the required intensity of clinical follow-up, encouraging adherence to therapy, and other approaches. Unfortunately, most clinical decision tools are focused primarily on their predictive ability at the expense of usability. Those developed to be usable, though, such as the $\mathrm{CHADS}_{2}$ score, ${ }^{8}$ sacrifice some predictive ability ${ }^{18-20}$ for ease of use. 
The Intermountain Mortality Risk Score (IMRS) is a sex-specific decision tool created to solve problems that inhibit risk score utilisation in clinical practice while maintaining a substantial ability to predict outcomes (ie, making them both usable and highly predictive).$^{21}$ IMRS addressed these challenges via data that are routinely collected or are inexpensive to obtain, and are electronically available from standardised, quantitative measurement methods. Furthermore, methods were applied to categorise variables into data-derived groupings (not overfit as continuous variables or ineffectively applied as clinically based thresholds). ${ }^{21}$ IMRS and similar 30-day readmission scores apply to heart failure (HF) and MI and eliminate the temporal cost to busy physicians by calculating the scores within an electronic health record (EHR) and providing them electronically, empowering clinicians to practise score-driven medicine without the burden of finding data or entering them into a risk calculator. ${ }^{22}$

This study sought to use the principles underlying IMRS to create usable but highly predictive Intermountain Major Adverse Cardiovascular Events (IMACE) risk scores for patients requiring long-term post-MI care, with the following purposes: (1) to predict MACE occurring 1-3 years after MI and (2) to be feasible for use in routine clinical care.

\section{METHODS \\ Study population}

The goal of this study was to develop a risk prediction tool to stratify long-term ( $>1$ year) post-MI risk. This risk tool would empower the determination of which patients have a favorable comparison of benefits vs. risks to whom enhanced MACE-ameliorating evaluation and care (including up to 36 months of DAPT) could be applied. Post-MI patients (aged $\geq 18$ years) admitted to Intermountain Medical Center, LDS Hospital or Cottonwood Hospital (Salt Lake City, Utah, USA) for an acute MI (defined as an MI by the attending cardiologist based on symptoms, ECG, and cardiac enzyme and troponin levels) during the era of P2Y 12 inhibitors (January 1997 through April 2014) were included as subjects. Subjects were followed until 30 April 2015. In keeping with the criteria of the Prevention of Cardiovascular Events in Patients with Prior Heart Attack Using Ticagrelor Compared to Placebo on a Background of Aspirin-Thrombolysis in Myocardial Infarction 54 (PEGASUS-TIMI 54) trial, inclusion was limited to patients with acute MI who survived $\geq 1$ year and had $\geq 1$ risk factor for recurrent MI (ie, age $\geq 65$ years, prior MI before baseline, $\geq 2$ vessel coronary artery disease, diabetes diagnosis or an estimated glomerular filtration rate $<60 \mathrm{~mL} / \mathrm{min} / 1.73 \mathrm{~m}^{2}$ ). Receipt of a $\mathrm{P}_{2} \mathrm{Y}_{12}$ inhibitor prescription was not required.

IMACE models were derived in $70 \%$ of subjects (women, n=1342; men, $n=3047$ ). For validation, IMACE equations were applied to the $30 \%$ who were held aside (women, $\mathrm{n}=576$; men, $\mathrm{n}=1290$ ). Assignment to derivation and validation used a pseudo-random number generator seeded by a long-period Mersenne Twister. A second validation population was composed of subjects free of MI at baseline and seen at the same hospitals who received percutaneous coronary intervention (PCI) with stent placement (women, $n=1779$; men, $n=4977$ ) during the same time frame.

\section{Risk score derivation}

IMACE was created based on the principles used previously for derivation of IMRS ${ }^{21}$ which has been validated repeatedly. ${ }^{21}{ }^{22}$ Its derivation used variables that are almost always available for most patients, making it possible to compute, adding little to no incremental financial expense, and empowering the delivery of IMACE to clinicians via the EHR such that clinical workflow is not interrupted. IMACE was formed from sex-specific multivariable Cox regression models using age, each factor in the complete blood count (CBC) and the comprehensive metabolic profile, troponin I, B-type natriuretic peptide (BNP), haemoglobin Alc (HbAlc), and the standard lipid panel. For further information about why only these variables were used, please see online supplementary methods regarding enhanced IMACE (e-IMACE) risk scores.

Relative weightings of each factor were determined from regression $\beta$-coefficients of multivariable Cox models that were evaluated for the composite primary MACE endpoint of cardiovascular (CV) mortality, MI or stroke. Inclusion of variables in Cox modelling used stepwise selection and forced entry based on results for non-missing data (among derivation patients, about $3 \%$ were missing CBC data, $2 \%$ basic metabolic profile parameters and $38 \%$ the additional six comprehensive metabolic profile factors, $20 \%$ lipid panel data, $68 \%$ BNP, $72 \%$ HbAlc, and 35\% troponin values). Mortality was determined from hospital records, Utah death certificates (including cause of death) and the national Social Security Death Master File, providing essentially complete follow-up for mortality events. ${ }^{23}$ Incident MI and stroke were determined based on International Classification of Diseases coding from Intermountain Healthcare electronic records of 22 hospitals throughout Utah and southern Idaho (Intermountain is the healthcare provider for approximately two-thirds of the population in these areas, and the vast majority of patients return to Intermountain facilities for subsequent care) that capture more than $90 \%$ of non-fatal events (lost to follow-up for non-fatal CV events is $<10 \%$ historically). ${ }^{23}$ In Cox regression, analyses were landmarked at 1 year, and censored at 3 years after the index MI for non-event subjects or at the time of mortality for deaths due to non-CV causes. Primary analyses used survival time until the first event for subjects with more than one MACE event.

Scalar variable weightings were applied to each independent variable to calculate IMACE using previously described methodology. ${ }^{21}$ Laboratory variables were categorised into quintiles plus a category for subjects with 
missing data. Age was categorised into decades. Some laboratory factors were excluded due to collinearity of variables: only one of the red blood cell count, haemoglobin and haematocrit triad was used, and one each from the following pairs: mean corpuscular volume/ mean corpuscular haemoglobin, sodium/chloride, creatinine/blood urea nitrogen (BUN), and total cholesterol/ low-density lipoprotein cholesterol. The Hosmer-Lemeshow statistic was used to evaluate model calibration.

Sex-specific thresholds for IMACE were established as follows: low risk was defined as IMACE values less than the threshold where sensitivity was $\geq 90 \%$ in the derivation population, high risk as scores equal to or above the threshold where specificity was $\geq 90 \%$, and mild and moderate risk as scores between those low-risk and highrisk thresholds, but further divided at the location where derivation results indicated a substantial increase in MACE risk from mild to moderate.

\section{Risk score validation}

Scalar scores for variables contributing to IMACE were applied to post-MI validation population to test whether IMACE applies outside of the derivation population. Cox regression was used with IMACE as the independent and MACE as the dependent variables. Both continuous and categorised IMACE variables were evaluated. HR, 95\% $\mathrm{CI}$ and $\mathrm{P}$ values are reported, along with percentages of events in IMACE risk categories.

Kaplan-Meier survival curves graphically examined the event-free survival by risk category and provided log-rank statistics. A P value of $\leq 0.05$ was designated as significant for sex-specific associations of IMACE with MACE in the validation population (ie, the derivation results were considered hypothesis-generating). Secondary endpoints were the individual events of MI, stroke, CV mortality and all-cause mortality, and required Bonferroni correction for five tests of hypothesis (these four plus the primary hypothesis) at $\mathrm{P} \leq 0.01$ for significance, with $\mathrm{P}>0.01$ but $\leq 0.05$ requiring further validation in future studies. Adjustment of IMACE associations was performed in Cox regression to evaluate the effect of adjusting for clinical variables not included as risk score components; further, e-IMACE was also created by adding other independent predictors such as HF diagnosis, atrial fibrillation history and medications (online supplementary methods).

\section{Further statistical considerations}

To compare the predictive ability of IMACE, the 6-month Global Registry of Acute Coronary Events (GRACE) score ${ }^{9-11} 1$-year IMRS ${ }^{21}$ and DAPT Study (DAPT-S) risk score $^{17}$ were calculated in the derivation and validation populations using previously published methodologies. Each risk score was compared with IMACE using measures of discrimination and improvement in risk stratification (ie, the c-statistics and net reclassification improvement (NRI) index) to determine to what extent IMACE provides additional value. ${ }^{24-27}$ Statistical significance for the NRI was assessed using an asymptotic test of a null hypothesis that NRI $=0 .{ }^{25-27}$ For IMACE, the low-risk and mild-risk groups were combined in assessments of the NRI, while IMRS ${ }^{21}$ and GRACE $^{28}$ categorisation used three standard groupings, and tertiles were used for DAPT-S scores. Both the DAPT-S full score that computes risk of events corrected for bleeding risk (by reducing the score for greater age) and the DAPT-S events-only score (excluding age) were calculated. ${ }^{17}$ The sensitivity, specificity, positive predictive value and negative predictive value (NPV) of each score were calculated for the highrisk group compared with all other groups.

Baseline characteristics are reported as percentages, means with SD, or medians with IQR, as appropriate. Comparisons of baseline variables between IMACE categories used the $\chi^{2}$ test, Student's t-test or analysis of variance, as appropriate. Continuous variables whose distribution violated the normality assumption were transformed using the natural log prior to analysis.

\section{RESULTS}

Variables evaluated for inclusion in IMACE are presented in online supplementary table S1 stratified by IMACE risk categories of low, mild, moderate and high risk: scores of $\leq 2,3-4,5-6$ and $\geq 7$ for women, and $\leq 2,3-5$, $6-7$ and $\geq 8$ for men, respectively. The maximum possible IMACE value was 12 for women but actually ranged from 0 to 11 , and was 16 for men with a range of $0-14$. Online supplementary table S2 presents the results for the variables used to create e-IMACE, including comorbidities and treatments. In derivation and validation groups, PCI was the mode of treatment for $58.7 \%$ and $55.4 \%$ of women and $66.2 \%$ and $67.3 \%$ of men, respectively. The average follow-up among non-event subjects was $2.9 \pm 0.4$ years in women and in men for both derivation and validation populations.

Among women, significant predictors of MACE were age, glucose, creatinine, haemoglobin, platelet count, red cell distribution width (RDW) and BNP. In men, MACE predictors were age, potassium, glucose, BUN, haematocrit, white blood cell count, RDW, mean platelet volume and BNP. Model calibration was good, with Hosmer-Lemeshow goodness of fit having $\mathrm{P}=0.79$ for women and $\mathrm{P}=0.55$ for men. Online supplementary table 3 displays the variable thresholds for each factor used to compose IMACE and the sample sizes of non-missing values, and reveals the substantial differences between predictor variables for women and men.

Survival curves for the association of IMACE with MACE demonstrated significant stratification in the validation groups for both women (figure 1A) and men (figure 1B). In women, comparisons between risk categories had log-rank $\mathrm{P}<0.001$ for IMACE moderate-risk versus low-risk and highrisk versus low-risk categories; men had $\mathrm{P}<0.001$ for both moderate-risk and high-risk versus low-risk categories (HRs and CIs are provided in online supplementary table 4). Among women, high-risk versus moderate-risk categories had $\log$-rank $\mathrm{P}=0.008$, with a trend towards significance for 
A)

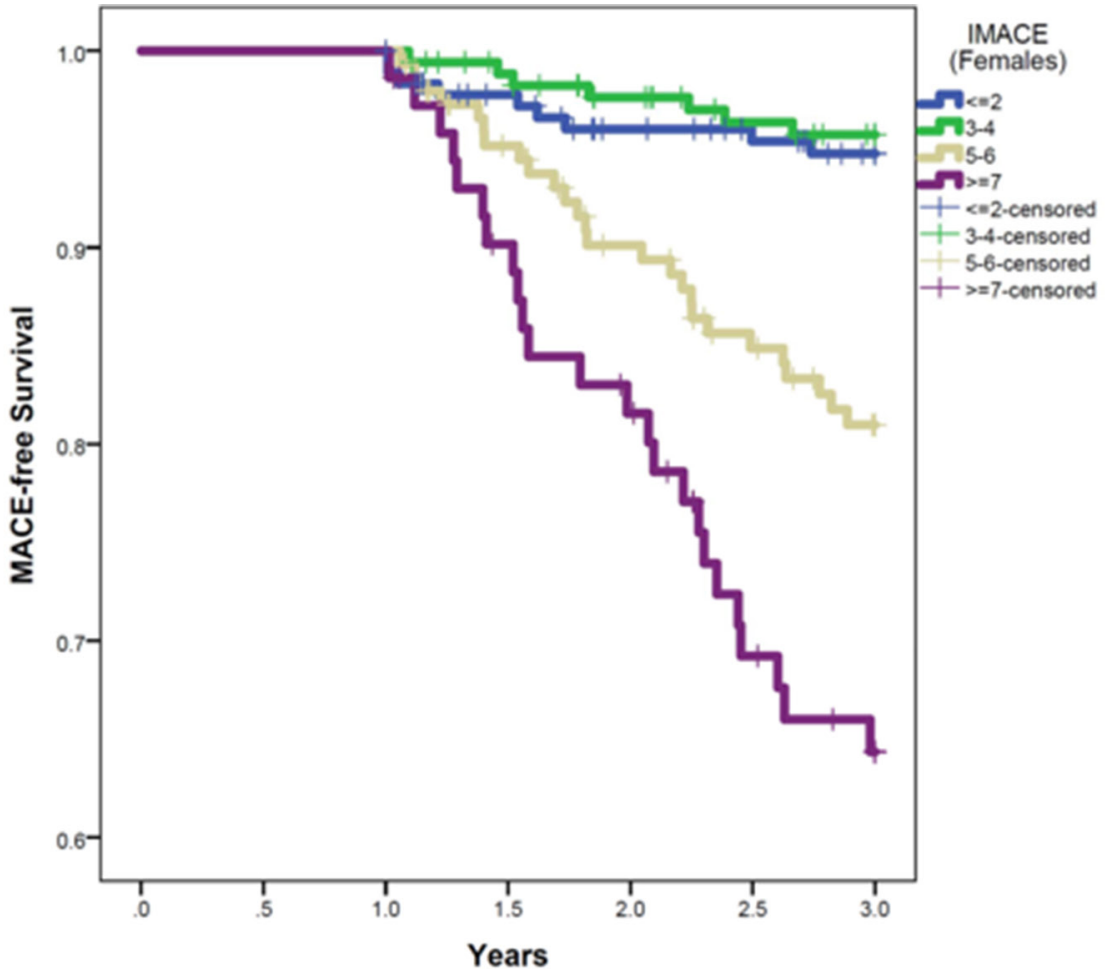

B)

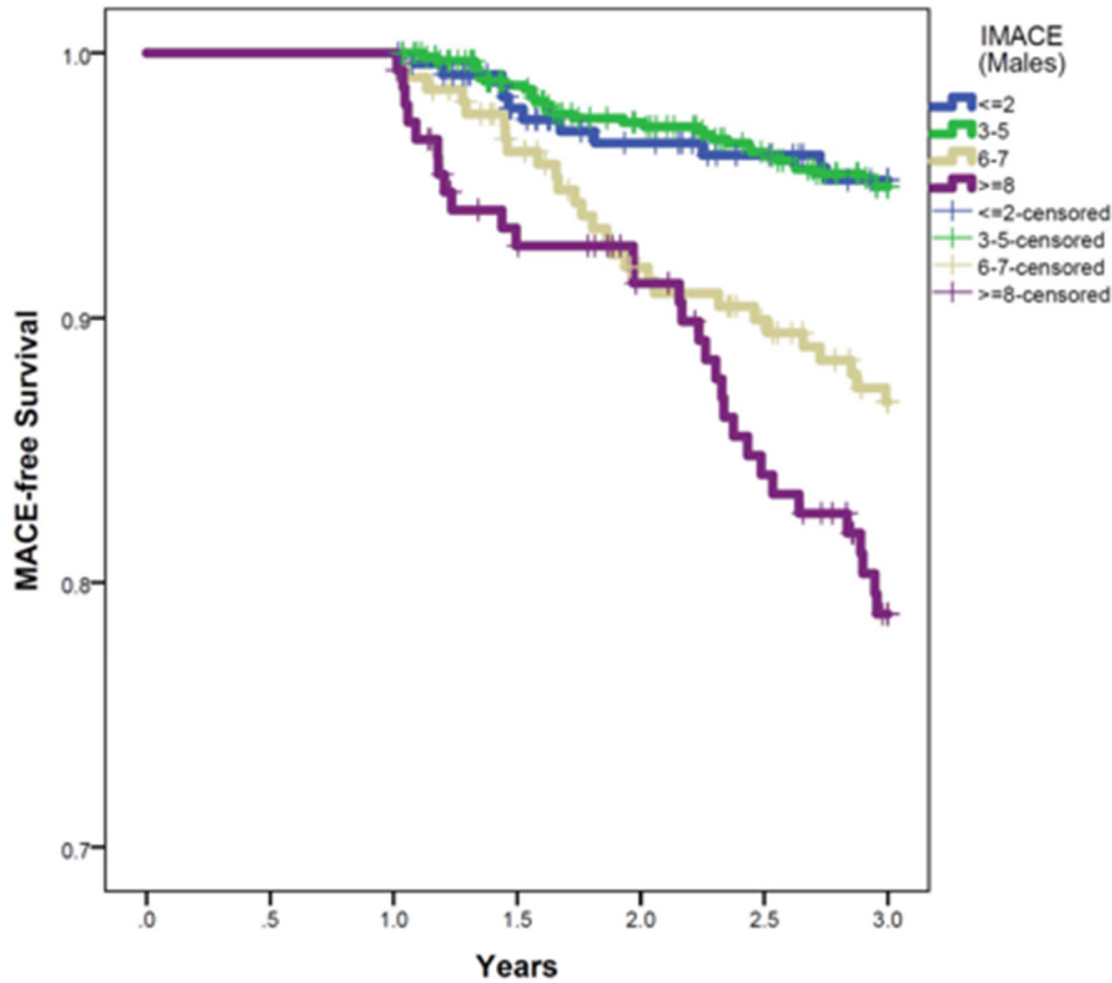

Figure 1 Kaplan-Meier survival curves showing associations of IMACE with MACE in the post-MI validation population: (A) women (log-rank $P$ trend $<0.001$ ) and (B) men (log-rank $P$ trend $<0.001$ ). IMACE, Intermountain Major Adverse Cardiovascular Events; MACE, major adverse cardiovascular events; MI, myocardial infarction.

that comparison in men $(\mathrm{P}=0.054$ for high-risk vs moderate-risk categories). The proportion of validation patients in each IMACE risk category for the composite outcome and individually for MI, stroke and CV mortality plus all-cause mortality is shown in table 1 . When modelled as a continuous variable, IMACE had $\mathrm{HR}=1.44$ per +1 score in women
(95\% CI 1.29 to $1.61 ; \mathrm{P}<0.001)$ and $\mathrm{HR}=1.29$ per +1 score in men (95\% CI 1.20 to $1.38 ; \mathrm{P}<0.001)$. HRs with $95 \%$ CI are shown in figure 2 for the validation population, both for IMACE alone and for models adjusting for factors from online supplementary table 2). Findings for the second validation population are shown in the online supplementary 
Table 1 Study MACE outcomes, 3 years after baseline MI, among women and men in the post-MI validation population

\begin{tabular}{|c|c|c|c|c|c|c|c|c|}
\hline \multicolumn{4}{|c|}{ Female } & & \multicolumn{4}{|l|}{ Male } \\
\hline$n=181$ & $n=174$ & $n=148$ & $n=73$ & & $n=247$ & $n=671$ & $n=218$ & $n=154$ \\
\hline \multicolumn{4}{|c|}{ IMACE risk category } & & \multicolumn{4}{|c|}{ IMACE risk category } \\
\hline Low & Mild & Mod & High & & Low & Mild & Mod & High \\
\hline$\leq 2(\%)$ & $3-4(\%)$ & 5-6 (\%) & $\geq 7(\%)$ & $\mathbf{P}$ trend & $\leq 2(\%)$ & $3-5(\%)$ & $6-7(\%)$ & $\geq 8(\%)$ \\
\hline
\end{tabular}

Study event

Primary outcome: MACE (composite of CV death, Ml and stroke)

\begin{tabular}{lrrrrrrrrrrrr}
$\quad$ Composite endpoint & 5.0 & 4.0 & 17.6 & 32.9 & $<0.001$ & 4.5 & 4.8 & 12.4 & 19.5 & $<0.001$ \\
Secondary outcomes & & & & & & & & & & \\
Ml & 1.7 & 1.1 & 4.1 & 9.6 & $<0.001$ & 2.8 & 2.5 & 6.0 & 5.8 & 0.012 \\
\hline Stroke & 0.6 & 0.0 & 2.7 & 4.1 & 0.005 & 0.0 & 0.3 & 2.8 & 0.6 & 0.016 \\
CV mortality & 2.8 & 3.4 & 12.2 & 21.9 & $<0.001$ & 1.6 & 2.4 & 6.0 & $14.3<0.001$ \\
\hline All-cause mortality & 3.9 & 4.6 & 16.9 & 30.1 & $<0.001$ & 2.8 & 3.3 & 8.3 & 17.5 & $<0.001$ \\
\hline
\end{tabular}

Sex-specific IMACE results are categorised as low, mild, moderate (mod) and high risk.

CV, cardiovascular; IMACE, Intermountain Major Adverse Cardiovascular Events; MACE, major adverse cardiovascular events;

$\mathrm{MI}$, myocardial infarction.

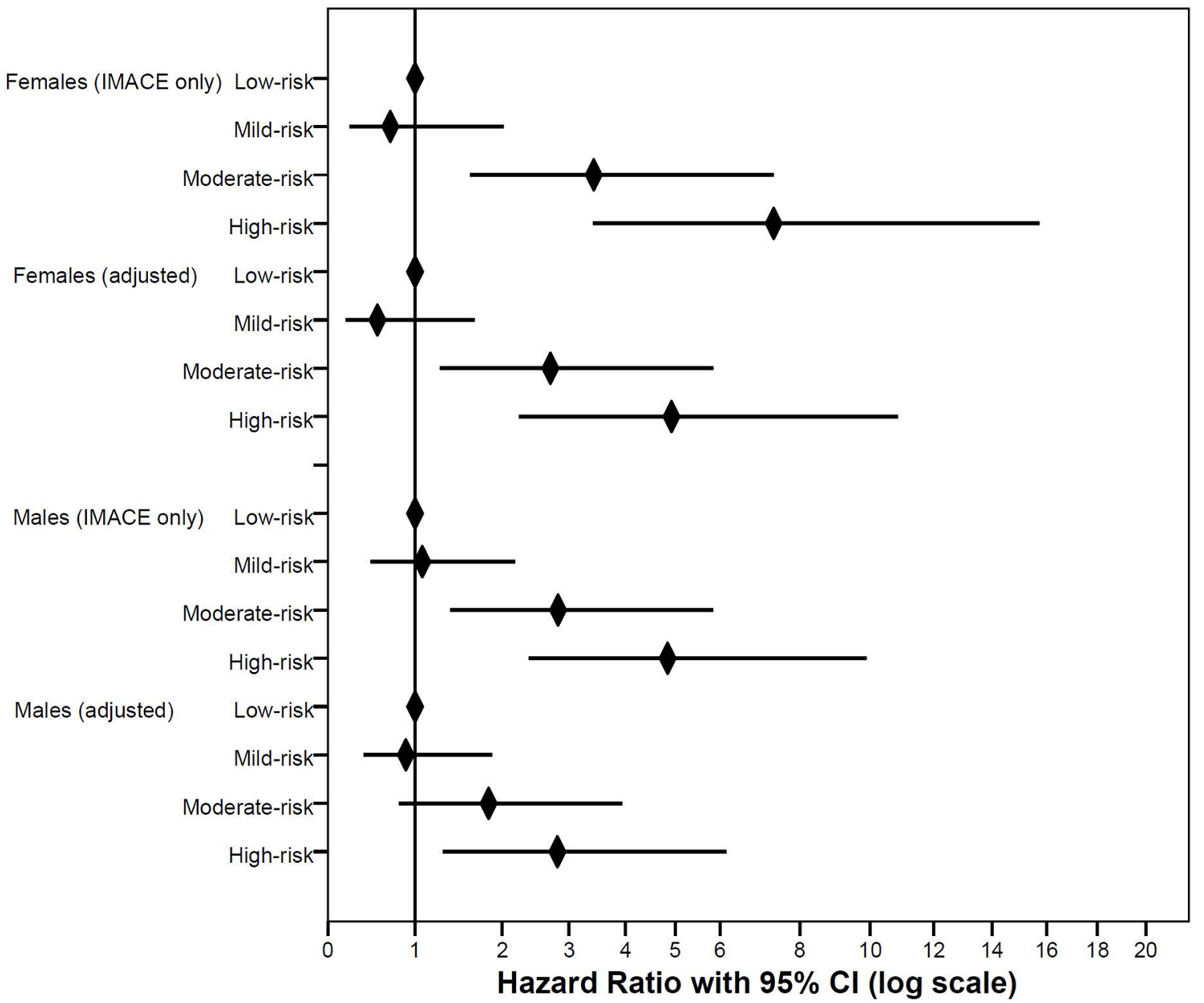

Figure $2 \mathrm{HR}(\mathrm{Cl})$ for the association of IMACE with MACE in women and men (post-MI validation), in univariable (IMACE only) and multivariable Cox regression adjusting for comorbidity and treatment variables (not included in IMACE: online supplementary table 2) and age (an IMACE component). IMACE, Intermountain Major Adverse Cardiovascular Events; MACE, major adverse cardiovascular events; MI, myocardial infarction. 
Table 2 Discrimination and reclassification statistics for the calculated values of the various risk scores that were studied, in both populations of post-MI subjects and for IMACE and DAPT-S in a secondary validation of non-MI poststent subjects

\begin{tabular}{|c|c|c|c|c|}
\hline Sex & Model & $\begin{array}{l}\text { Derivation } \\
\text { c-statistics }\end{array}$ & $\begin{array}{l}\text { Validation } \\
\text { c-statistics }\end{array}$ & $\begin{array}{l}\text { NRI index for } \\
\text { IMACE vs } \\
\text { the indicated } \\
\text { score }\end{array}$ \\
\hline \multirow[t]{7}{*}{ Female } & & $n=1342$ & $\mathrm{n}=576$ & \\
\hline & IMACE & 0.675 & 0.734 & - \\
\hline & e-IMACE & 0.717 & 0.717 & $\mathrm{~N} / \mathrm{C}$ \\
\hline & IMRS & 0.652 & 0.690 & $\begin{array}{l}0.478 \\
(\mathrm{P}<0.001)\end{array}$ \\
\hline & GRACE & 0.603 & 0.632 & $\begin{array}{l}0.282 \\
(P<0.001)\end{array}$ \\
\hline & DAPT-S & 0.546 & 0.574 & $\mathrm{~N} / \mathrm{C}$ \\
\hline & $\begin{array}{l}\text { DAPT-S no } \\
\text { age }\end{array}$ & 0.606 & 0.649 & $\begin{array}{l}0.205 \\
(P<0.001)\end{array}$ \\
\hline \multirow[t]{7}{*}{ Male } & & $n=3047$ & $n=1290$ & \\
\hline & IMACE & 0.715 & 0.672 & - \\
\hline & e-IMACE & 0.734 & 0.667 & $\mathrm{~N} / \mathrm{C}$ \\
\hline & IMRS & 0.633 & 0.629 & $\begin{array}{l}0.250 \\
(P<0.001)\end{array}$ \\
\hline & GRACE & 0.649 & 0.670 & $\begin{array}{l}0.127 \\
(\mathrm{P}<0.001)\end{array}$ \\
\hline & DAPT-S & 0.581 & 0.551 & $\mathrm{~N} / \mathrm{C}$ \\
\hline & $\begin{array}{l}\text { DAPT-S, no } \\
\text { age }\end{array}$ & 0.661 & 0.628 & $\begin{array}{l}0.157 \\
(P<0.001)\end{array}$ \\
\hline \multicolumn{5}{|c|}{$\begin{array}{l}\text { Second validation population of non-Ml poststent subjects (women, } \\
n=1779 ; \text { men, } n=4977 \text { ) }\end{array}$} \\
\hline \multirow[t]{3}{*}{ Female } & IMACE & NA & 0.635 & - \\
\hline & DAPT-S & NA & 0.510 & $\mathrm{~N} / \mathrm{C}$ \\
\hline & $\begin{array}{l}\text { DAPT-S, no } \\
\text { age }\end{array}$ & NA & 0.581 & $\begin{array}{l}0.044 \\
(P<0.001)\end{array}$ \\
\hline \multirow[t]{3}{*}{ Male } & IMACE & NA & 0.635 & - \\
\hline & DAPT-S & NA & 0.553 & $\mathrm{~N} / \mathrm{C}$ \\
\hline & $\begin{array}{l}\text { DAPT-S, no } \\
\text { age }\end{array}$ & NA & 0.619 & $\begin{array}{l}0.026 \\
(\mathrm{P}<0.001)\end{array}$ \\
\hline
\end{tabular}

NRI index was calculated for validation subjects. DAPT, dual antiplatelet therapy; DAPT-S, DAPT Study; e-IMACE, enhanced IMACE; GRACE, Global Registry of Acute Coronary Events; IMACE, Intermountain Major Adverse Cardiovascular Events; IMRS, Intermountain Mortality Risk Scores; MI, myocardial infarction; NA, not applicable; N/C, not calculated; NRI, net reclassification improvement.

results. Association of IMACE with the composite outcome in ST elevation MI versus non-ST elevation MI did not differ, with formal test of interaction showing P interaction $=0.19$ in validation women and $P$ interaction $=0.45$ in validation men.

IMACE and other risk score c-statistics in the derivation and validation populations are shown in table 2 (see online supplementary figures S1-S3 for the IMRS, GRACE and DAPT-S Kaplan-Meier survival curves). For women, IMACE had $90 \%$ specificity and $96 \%$ NPV, while for men it had 90\% specificityand 95\% NPV. Other measures of predictive value and those of the IMRS, GRACE and DAPT-S risk scores are found in online supplementary table S5. For IMACE, accuracy in the derivation and primary validation groups was $82.1 \%$ and $84.2 \%$ for women and $84.1 \%$ and $85.0 \%$ for men, respectively. IMACE significantly reclassified risk stratification of MACE for women and men compared with IMRS, GRACE and DAPT-S risk scores (table 2).

\section{DISCUSSION}

\section{Study findings}

Sex-specific risk scores were created in stable post-MI patients ( $\geq 1$ year free from CV events) with $\geq 1$ risk factor for recurrent MI. IMACE equations were validated to predict MACE in an independent set of post-MI patients and a second validation of non-MI stent recipients. These dynamic EHR-delivered scores performed well compared with other risk scores. IMACE differentiates itself also in being an easily implemented EHR-based tool that summarises risk using common tests that post-MI patients receive routinely and serially. Developed in part based on previously implemented clinical risk stratification methods, ${ }^{22}$ IMACE provides additional information to clinicians to facilitate the best use of their expertise in clinical decision making without adding burden to their workflow.

\section{Clinical context}

Various studies have evaluated extended DAPT beyond 1 year of therapy. DAPT-S showed that, compared with aspirin alone, clopidogrel $(75 \mathrm{mg} /$ day $)$ or prasugrel $(10 \mathrm{mg} /$ day $)$ with aspirin reduced stent thrombosis risk by $71 \%$ and a composite of all-cause mortality, MI or stroke by $29 \%$ in subjects receiving drug-eluting stents. ${ }^{13}$ Risk of bleeding was increased by DAPT. ${ }^{13}$ The Optimal Dual Antiplatelet Therapy trial found a non-significant $(\mathrm{P}=0.17)$ but similar magnitude $25 \%$ reduction in risk of events for long-term DAPT, with no difference in bleeding risk. ${ }^{14}$

The PEGASUS-TIMI 54 trial provided the evidence base for expansion of the Food and Drug Administration-approved ticagrelor indication to include a twicedaily $60 \mathrm{mg}$ dose (with 75-150 mg of aspirin daily) beyond 1 year post-MI. ${ }^{12}$ This trial included patients with an MI 1-3 years prior to enrolment and found significant reduction in MACE for long-term DAPT, at the cost of higher rates of major bleeding. ${ }^{12}$ Given this and other evidence of a modest but important benefit from long-term DAPT above the mild increase in bleeding risk, ${ }^{15}$ revised guidelines for $>1$ year of DAPT were released in $2016 .{ }^{16}$ In particular, they reiterate the need to balance DAPT's long-term CV benefit with the risk of major bleeding.

\section{Clinical application}

To quantify the benefits and costs of long-term DAPT, the DAPT-S risk score was created to identify patients whose potential DAPT benefit is greater than its bleeding risk. ${ }^{17}$ In contrast to the IMACE derivation population, the DAPT-S score was created among subjects who received PCI with drug-eluting stents and explicitly models both benefits and risks of DAPT. In a validation 
population, the DAPT-S score discrimination of risk for MI or stent thrombosis was modest $(c=0.64) .{ }^{17}$ In the present study's post-MI validation, it was poorly predictive of MACE $(\mathrm{c}<0.60$; table 2$)$, but this was improved by removing the DAPT-S score's age-based correction for bleeding risk (men: $\mathrm{c}=0.60$, women: $\mathrm{c}=0.65$ ). In the same post-MI validation, IMACE had $\mathrm{c}=0.734$ for women $(+0.085$ to +0.160 greater than the DAPT-S scores $)$ and $\mathrm{c}=0.672$ for men (+0.044 to +0.121 greater than the DAPT-S score), with IMACE NRIs well above the de facto standard of 0.10 .

IMACE, like IMRS, was created for broad application to all types of enhanced evaluation and care, including by harnessing the risk information from emerging clinical risk factors like the RDW. ${ }^{212} 2930$ IMACE was derived in patients who were not on long-term DAPT; consequently, it does not explicitly model bleeding risk. IMACE can be applied to weighing the risks and benefits of long-term DAPT given its ability to stratify risk of MACE, but also to the costs and benefits of other clinical decisions that may be considered in a patient's long-term care plan, such as greater frequency of follow-up visits, application of remote health monitoring and electronic nudging to increase compliance with medications and health interventions, and more intense long-term dosing of other post-MI medications.

Altering a patient care plan for reduction of $\mathrm{CV}$ events, however, only begins with a precision risk score in a learning healthcare system. The optimal approach combines the risk score with implementation science, including a method of delivery that does not require a physician or staff member's involvement to obtain the risk information and a care process model (CPM) that is attuned to a patient's risk stratum. Previously, a successful marriage was performed of usable EHR-based risk scores (ie, IMRS and an Intermountain 30-day readmission risk score for patients with HF) to a CPM derived for patients with higher risk $\mathrm{HF}^{22}$ Inpatients with $\mathrm{HF}$ with a high risk of 30-day readmission or of 30-day mortality were managed according to the high-intensity CPM (lower risk patients received standard care). Pilot results showed improved 30 -day mortality ( $7 \%$ after implementation vs $19 \%$ preimplementation), with no increase in readmission $(12.8 \%$ after vs $14.1 \%$ preimplementation (with denominators corrected for those alive and at risk of readmission)). ${ }^{22}$ Development of the CPM was performed by clinicians using published evidence and best care practices. ${ }^{22}$

Using a similar methodology to the HF programme, ${ }^{22}$ IMACE can be used in conjunction with IMRS or other scores that predict risk during the first post-MI year and are either fully or partially integrated on the back end of an EHR, where the data processing is outside of the clinical environment but can be then delivered seamlessly to clinicians. IMACE would be used among patients likely to survive that first year to identify who will remain or transition to higher risk in years 1-3 (ie, IMACE score in the moderate or high group) and for whom long-term (>1 year) therapies may provide greater absolute benefit than harm (patients with low or mild risk IMACE scores would likely derive less absolute MACE-reducing benefit and should receive standard care). That is, a higher risk IMACE score could trigger additional evaluations or services at index hospitalisation and during the first year after MI since this long-term post-MI score reveals higher risk that should be ameliorated as soon as possible. Serial IMACE testing (eg, at 1 year post-MI) may also aid in patient evaluation and planning for post-MI years 1-3 because the common laboratory tests underlying IMACE are dynamic, unlike comorbidities or histories of adverse events or procedures, and thus IMACE when measured serially may provide important information about increasing or decreasing risk over time. ${ }^{29}$

For example, a baseline evaluation of and planning for impacting a patient's social risk factors (eg, schedule routine patient contact or provide methods for the patient to access an advanced practice clinician rather than just going to the emergency department) would be valuable given the potential continuing adverse impact of these over the long term. A baseline education session by a pharmacist to better instruct the patient about their reconciled list of medications or by an advanced practice clinician regarding other health education needs prior to discharge could be considered and more frequent (than standard) clinic visits during the first year after MI could be valuable. Deployment of higher cost remote health monitoring and electronic nudging to increase compliance with medications and health interventions may be justified for those who will survive for $>1$ year post-MI but will have potentially more morbidities and health system encounters as indicated by higher IMACE score. Also, a higher IMACE could motivate clinicians to aggressively ensure that the patient attends their 1-year post-MI clinic visit to reassess risk scores (previously we showed that yearly recalculation of IMRS provided added prognostic information ${ }^{29}$ ) and biomarkers, and to re-evaluate patient health education, medication needs and compliance, and success of risk factor reduction.

\section{Limitations}

The DAPT-S risk score ${ }^{17}$ GRACE model $^{9-11}$ and IMRS ${ }^{21}$ were compared with IMACE herein (see online supplementary dicussion), but were created to predict different endpoints or for distinct follow-up periods. Study comparisons may consequently be imperfect and interpretation of results should be performed with caution. At least one implication that is clear, though, is that risk scores created for prognostication during the first year after MI are not as applicable to post-MI years 1-3 as scores created specifically for that long-term period.

Inclusion of subjects for derivation and validation of IMACE was limited to patients who were event-free for 1 year after index MI. Consequently, the setting for application of IMACE is among post-MI patients, although application to non-MI PCI patients herein showed substantial value for applying IMACE. Usefully, IMACE was composed using data available at baseline; thus, 
modification of a patient care plan based on IMACE can be performed with a perspective on long-term patient health. Further, while the Intermountain data registry used to derive and validate IMACE compares favourably with other international CV populations such as the Reduction of Atherothrombosis for Continued Health and the Study of Heart and Renal Protection registries, ${ }^{3132}$ some factors such as smoking and race are substantially different. Subjects included here were from one geographical region, and additional replications of IMACE in geographically and racially distinct populations are required; however, because IMRS was created in a population from the same region and replicated in populations from various locations across the world, ${ }^{21} 3033$ it is anticipated that IMACE will replicate in other populations as well.

While ST-elevation MI (STEMI) data were included in e-IMACE, other ECG data were unavailable in this study but would be valuable to consider in future evaluations of IMACE. Due to the passive surveillance methods for capturing non-fatal health events, findings herein may be biased by misspecification of MACE status, although this should only bias towards the null hypothesis. Finally, IMACE does not explicitly account for the bleeding risk from DAPT; therefore, any CPM created to guide care should explicitly account for this.

\section{CONCLUSIONS}

IMACE sex-specific risk scores effectively stratified 1-year to 3-year post-MI risk of CV events with modest (men) to excellent (women) risk discrimination. IMACE is an inexpensive, efficient, dynamic EHR-delivered tool that empowers the evaluation of which post-MI patients may be the best candidates for more aggressive long-term therapeutic management.

Contributors BDH, JBM and DLL designed the study. BDH, JBM, DB, GLH, NDK and TLB planned the execution of the study. BDH and TLB collected and prepared the data. BDH conducted statistical analyses. BDH, JBM, DB, GLH, NDK and DLL evaluated and interpreted the results. BDH and DLL drafted the manuscript, and JBM, $\mathrm{DB}, \mathrm{GLH}, \mathrm{NDK}, \mathrm{TLB}$ and DLL revised the paper for important intellectual content.

Funding This work was supported by an unrestricted educational grant from AstraZeneca to $\mathrm{BDH}, \mathrm{JBM}$ and $\mathrm{DLL}$, and these Intermountain investigators had final authority over manuscript content.

Competing interests BDH is an inventor of IMRS and two readmission risk scores that are licensed to CareCentra for integration into their products. BDH is Pl of grants funded by Intermountain Healthcare's Foundry Innovation Program, the Intermountain Research and Medical Foundation, CareCentra, AstraZeneca and GlaxoSmithKline for the development and/or implementation of clinical risk scores. DB, GLH and NDK are employees of AstraZeneca.

Ethics approval This study was approved by the Intermountain Healthcare Institutional Review Board and was determined to be a minimal risk, general dataonly study that posed minimal risk to subjects and for which a waiver of consent was provided.

Provenance and peer review Not commissioned; externally peer reviewed.

Data sharing statement While the data used in this study are HIPAA-protected private information, with the execution of an appropriate institutional contractual agreement, the authors are pleased to collaborate with other scientists on further evaluations of the study data or the larger source data sets available through Intermountain Healthcare.
Open Access This is an Open Access article distributed in accordance with the Creative Commons Attribution Non Commercial (CC BY-NC 4.0) license, which permits others to distribute, remix, adapt, build upon this work non-commercially, and license their derivative works on different terms, provided the original work is properly cited and the use is non-commercial. See: http://creativecommons.org/ licenses/by-nc/4.0/

(C) Article author(s) (or their employer(s) unless otherwise stated in the text of the article) 2018. All rights reserved. No commercial use is permitted unless otherwise expressly granted.

\section{REFERENCES}

1. Fine MJ, Auble TE, Yealy DM, et al. A prediction rule to identify lowrisk patients with community-acquired pneumonia. $N$ Engl J Med 1997;336:243-50.

2. Wilson PW, D'Agostino RB, Levy D, et al. Prediction of coronary heart disease using risk factor categories. Circulation 1998;97:1837-47.

3. Morrow DA, Antman EM, Charlesworth A, et al. TIMI risk score for ST-elevation myocardial infarction: A convenient, bedside, clinical score for risk assessment at presentation: An intravenous nPA for treatment of infarcting myocardium early II trial substudy. Circulation 2000;102:2031-7.

4. Sianos G, Morel MA, Kappetein AP, et al. The SYNTAX Score: an angiographic tool grading the complexity of coronary artery disease. Eurolntervention 2005;1:219-27.

5. Rothwell PM, Giles MF, Flossmann E, et al. A simple score (ABCD) to identify individuals at high early risk of stroke after transient ischaemic attack. Lancet 2005;366:29-36.

6. Halkin A, Singh M, Nikolsky E, et al. Prediction of mortality after primary percutaneous coronary intervention for acute myocardial infarction: the CADILLAC risk score. J Am Coll Cardiol 2005;45:1397-405.

7. Ketchum ES, Dickstein K, Kjekshus J, et al. The Seattle Post Myocardial Infarction Model (SPIM): prediction of mortality after acute myocardial infarction with left ventricular dysfunction. Eur Heart J Acute Cardiovasc Care 2014;3:46-55.

8. Gage BF, Waterman AD, Shannon W, et al. Validation of clinical classification schemes for predicting stroke: results from the National Registry of Atrial Fibrillation. JAMA 2001;285:2864-70.

9. Granger CB, Goldberg RJ, Dabbous O, et al. Predictors of hospital mortality in the global registry of acute coronary events. Arch Intern Med 2003;163:2345-53.

10. Fox KA, Dabbous OH, Goldberg RJ, et al. Prediction of risk of death and myocardial infarction in the six months after presentation with acute coronary syndrome: prospective multinational observational study (GRACE). BMJ 2006;333:1091-4.

11. Kaa F, Fitzgerald G, Puymirat E, et al. Should patients with acute coronary syndromes be stratified for management according to their risk? BMJ Open 2014;4:e004425.

12. Bonaca MP, Bhatt DL, Cohen M, et al. Long-term use of ticagrelor in patients with prior myocardial infarction. $N$ Engl J Med 2015;372:1791-800.

13. Mauri L, Kereiakes DJ, Yeh RW, et al. Twelve or 30 months of dual antiplatelet therapy after drug-eluting stents. N Engl J Med 2014;371:2155-66.

14. Helft G, Steg PG, Le Feuvre C, et al. Stopping or continuing clopidogrel 12 months after drug-eluting stent placement: the OPTIDUAL randomized trial. Eur Heart J 2016;37:365-74.

15. Bittl JA, Baber U, Bradley SM, et al. Duration of dual antiplatelet therapy: A systematic review for the 2016 ACC/AHA guideline focused update on duration of dual antiplatelet therapy in patients with coronary artery disease: A report of the American College of Cardiology/American Heart Association task force on clinical practice guidelines. Circulation 2016;134:e156-78.

16. Levine GN, Bates ER, Bittl JA, et al. ACC/AHA guideline focused update on duration of dual antiplatelet therapy in patients with coronary artery disease. Circulation 2016;2016:e123-55.

17. Yeh RW, Secemsky EA, Kereiakes DJ, et al. Development and validation of a prediction rule for benefit and harm of dual antiplatelet therapy beyond 1 year after percutaneous coronary intervention. JAMA 2016;315:1735-49.

18. Chen JY, Zhang AD, Lu HY, et al. CHADS2 versus CHA2DS2VASc score in assessing the stroke and thromboembolism risk stratification in patients with atrial fibrillation: a systematic review and meta-analysis. J Geriatr Cardiol 2013;10:258-66.

19. Van Staa TP, Setakis E, Di Tanna GL, et al. A comparison of risk stratification schemes for stroke in 79,884 atrial fibrillation patients in general practice. J Thromb Haemost 2011;9:39-48. 
20. van den Ham HA, Klungel OH, Singer DE, et al. Comparative performance of ATRIA, CHADS2, and CHA2DS2-VASc risk scores predicting stroke in patients with atrial fibrillation: results from a national primary care database. J Am Coll Cardiol 2015;66:1851-9.

21. Horne BD, May HT, Muhlestein JB, et al. Exceptional mortality prediction by risk scores from common laboratory tests. Am J Med 2009;122:550-8.

22. Evans RS, Benuzillo J, Horne BD, et al. Automated identification and predictive tools to help identify high-risk heart failure patients: pilot evaluation. J Am Med Inform Assoc 2016;23:872-8.

23. Muhlestein JB, Horne BD, Bair TL, et al. Usefulness of in-hospital prescription of statin agents after angiographic diagnosis of coronary artery disease in improving continued compliance and reduced mortality. Am J Cardiol 2001;87:257-61.

24. Pencina MJ, D'Agostino RB, D'Agostino RB, et al. Evaluating the added predictive ability of a new marker: from area under the ROC curve to reclassification and beyond. Stat Med 2008;27:157-72.

25. Pencina MJ, D'Agostino RB, Steyerberg EW. Extensions of net reclassification improvement calculations to measure usefulness of new biomarkers. Stat Med 2011;30:11-21.

26. Pencina MJ, D'Agostino RB, Vasan RS. Statistical methods for assessment of added usefulness of new biomarkers. Clin Chem Lab Med 2010;48:1703-11.
27. Leening MJG, Vedder MM, Witteman JCM, et al. Net reclassification improvement: computation, interpretation, and controversies. Ann Intern Med 2014;160:122-31.

28. GRACE ACS Risk Score. http://gracescore.co.uk/risk-stratification (accessed 3 Sep 2016).

29. Horne BD, Lappé DL, Muhlestein JB, et al. Repeated measurement of the intermountain risk score enhances prognostication for mortality. PLoS One 2013;8:e69160.

30. Horne BD, Anderson JL, Muhlestein JB, et al. Complete blood count risk score and its components, including RDW, are associated with mortality in the JUPITER trial. Eur J Prev Cardiol 2015;22:519-26.

31. Bhatt DL, Steg PG, Ohman EM, et al. International prevalence, recognition, and treatment of cardiovascular risk factors in outpatients with atherothrombosis. JAMA 2006;295:180-9.

32. Baigent C, Landray MJ, Reith C, et al. The effects of lowering LDL cholesterol with simvastatin plus ezetimibe in patients with chronic kidney disease (Study of Heart and Renal Protection): a randomised placebo-controlled trial. Lancet 2011;377:2181-92.

33. Rödel AP, Sangoi MB, de Paiva LG, et al. Complete blood cell count risk score as a predictor of in-hospital mortality and morbidity among patients undergoing cardiac surgery with cardiopulmonary bypass. Int J Cardiol 2015;187:60-2. 